\title{
MC-CDMA based SDR for Next Generation Wireless Communication
}

\author{
T. Ilavarasi \\ PG Scholar \\ Sri Venkateswara College of \\ Engineering, Pennalur, \\ Chennai-602117, Tamil Nadu
}

\author{
N. Kumaratharan \\ Professor \\ Sri Venkateswara College of \\ Engineering, Pennalur, \\ Chennai-602117, Tamil Nadu
}

\author{
K. Rasadurai \\ Research Scholar \\ Sri Venkateswara College of \\ Engineering, Pennalur, \\ Chennai-602117, Tamil Nadu
}

\begin{abstract}
Recent innovations in wireless technology have made a need to propose hybrid systems to achieve excellent data rates and performance. One such system is multi-carrier code division multiple access (MC-CDMA), a combination of direct sequence code division multiple access (DS-CDMA) and orthogonal frequency division multiplexing (OFDM) systems. It offers high data rate, flexible bandwidth allocation and enhanced performance to each user simultaneously. Owing to these advantages, this system is chosen to be interfaced with the physical layer of software defined radio (SDR). SDR is defined as a reprogrammable hardware that suits emerging standards of next generation wireless communication. In MC-CDMA system, when the orthogonality is not maintained in the OFDM part, it leads to multiple access interference (MAI). To overcome the effects of MAI and to reduce complex detection to a great extent, super orthogonal turbo codes is chosen for the system. In this paper, MC-CDMA based SDR system is constructed using super orthogonal turbo codes with log-MAP algorithm. The simulation results show that the performance is remarkable when compared to a turbo system using recursive systematic codes (RSC). The BER performance of both the systems is analyzed for different iterations.
\end{abstract}

\section{KEYWORDS}

MC-CDMA, SDR, SOTC, RSC and MUD

\section{INTRODUCTION}

The major goal of $4 \mathrm{G}$ is the integration of existing systems through a generic architecture. This would be feasible if there is a reprogrammable hardware like SDR. SDR is simply defined as a transceiver, where functions are realized as programs on re-programmable components. It offers compatibility between existing wireless networks and hence it is open to adopt new technologies. Thus it plays an important role in the success and penetration of $4 \mathrm{G}$ systems. MC-CDMA belongs to $4 \mathrm{G}$ which is a combination of CDMA and OFDM that inherits the benefits of both the systems. It is chosen to be interfaced in the physical layer of SDR to provide excellent performance and good quality of service

(QoS) to each user. Hence MC-CDMA based SDR system would be a well-built candidate that supports existing and future standards.

The $4^{\text {th }}$ generation mobile communication is expected to offer $100 \mathrm{Mbps}$ or higher data rates in downlink. MC-CDMA and OFDM are potential candidates [1] for next generation mobile communication. The performance of CDMA adaptable with SDR is proved [2] to provide high data rates, yet CDMA suffers from multipath fading with increased number of users. As an attempt MC-CDMA system is made adaptable to SDR since it overcomes the effects of multipath fading and thereby satisfies multiple users at the same time. However, it suffers from multiple access interference (MAI) and complex detection. MAI affects the BER performance in MC-CDMA system; hence to mitigate it, joint multiuser detection (MUD) and Turbo decoding [3] are used to improve the system performance. Among all turbo MUD techniques, maximum likelihood (ML) MUD shows better performance [4] by using log-MAP algorithm. Turbo codes are parallel concatenated codes that shown to be near the Shannon's theoretical limit [5]. Super orthogonal turbo codes (SOTC) are low-rate convolutional codes [6,7] chosen since it outperforms other existing codes and reduces complex detection to a great extent. However, the complexity of decoding increases exponentially as the number of users increases [8] and to reduce this problem, MUD techniques such as parallel and serial interference cancellation (PIC \& SIC), general minimum mean square error (GMMSE) and polynomial MMSE has been proposed. Signal detection schemes [9] such as minimum mean square error (MMSE), zero forcing (ZF), sphere decoding (SD), Q-less QR decomposition are studied for different modulations to analyze system performance. From the available coding techniques and MUD detection methods, a MC-CDMA based SDR using super orthogonal turbo codes [10] with log-MAP algorithm is constructed and simulated for different iterative values. 
The paper is organized as follows: Section II describes the system to be constructed. The importance of using SOTC codes is detailed in section III. Maximum a posteriori algorithm is explained in section IV. Simulation results and discussions are presented in section $\mathrm{V}$ and the conclusion is arrived at section VI.

\section{SYSTEM MODEL}

\subsection{MC-CDMA based SDR Transmitter}

SDR consists of three layers; namely, physical layer, data link layer and application layer. The physical layer is enabled with existing systems so that it becomes adaptable to SDR technology. MC-CDMA system is made adaptable to SDR [1] to support future wireless communication systems. The data signal for MC-CDMA is generated by combining CDMA and OFDM systems. The input data signal is spreaded using a spreading sequence followed by inverse fast fourier transform (IFFT) modulation to confirm the orthogonality among the entire signals for excellent transmission with least interference. After passing through the channel, the received signal is down converted and demodulated followed by efficient detection to deliver the appropriate signal to all users.

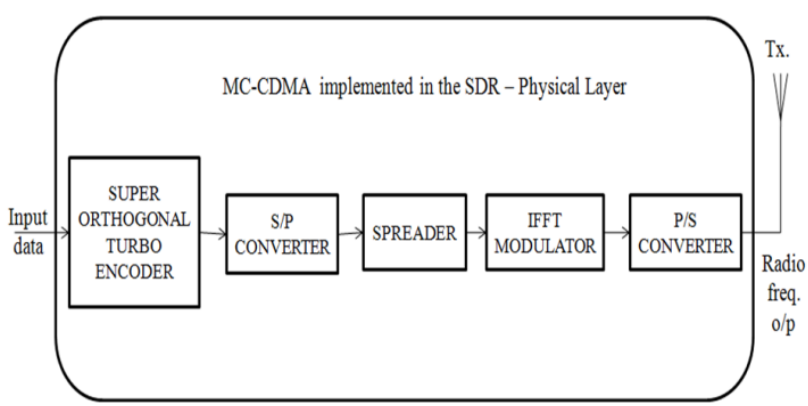

Fig-1: MC-CDMA based SDR Transmitter

The input data along with Hadamard matrix is encoded using SOTC code. The principle of DS-CDMA is to spread the encoded data symbol with a spreading code $c^{(k)}(t)$ of length $L$,

$$
c^{(k)}(t)=\sum_{l=0}^{L-1} c_{l}^{(k)} p T_{c}\left(t-l T_{c}\right)
$$

assigned to user $k, k=0, \ldots, K-1$, where $K$ be the total number of active users. After spreading, the encoded signal $x^{(k)}(t)$ of user $k$ is given by

$$
x^{(k)}(t)=d^{(k)} \sum_{l=0}^{L-1} c_{l}^{(k)} p T_{c}\left(t-l T_{c}\right), \quad 0 \leq t<T_{d}
$$

for each data symbol duration $T_{d}=L T_{c}$, where $T_{c}$ is the chip duration, $d(k)$ be the transmitted data symbol of user $k$. The multiplication of the information sequence with the spreading sequence is done bit-synchronously and the overall transmitted signal $x(t)$ of all $k$ synchronous users results in

$$
x(t)=\sum_{k=0}^{K-1} x^{(k)}(t)
$$

Since MAI strongly depends on cross-correlation function (CCF) of the spreading sequences, the CCF should be as small as possible. These multiple sub-carriers follow OFDM modulation with spacing of symbol frequency $F_{S}$

$$
F_{S}=\frac{1}{T_{s}}
$$

in order to achieve orthogonality among the signals. However, to completely avoid the effects of ISI and thus, to maintain orthogonality, a guard interval of duration is given by

$$
T_{g} \geq \tau_{\max }
$$

The guard interval is a cyclic extension of each OFDM symbol by extending the duration of an OFDM symbol to

$$
T_{s}^{\prime}=T_{g}+T_{s}
$$

The signal $x(t)$ after being upconverted and the RF signal is transmitted to the channel.

\subsection{MC-CDMA based SDR Receiver}

The output of the channel, after RF down conversion is the received signal $y(t)$ obtained from the convolution of $x(t)$ with impulse response $h(t)$ and addition of a noise signal $n(t)$ as follows,

$$
\begin{aligned}
y(t) & =x(t) * h(t)+n(t) \\
& =\sum_{k=0}^{K-1} r^{(k)}(t)+n(t)
\end{aligned}
$$

where $r^{(k)}(t)=x^{(k)}(t)^{*} h(t)$ be the noise-free received signal of user $k, n(t)$ be the additive white Gaussian noise (AWGN) and * denotes the convolution operation. After doing analog to digital conversion of the received signal $y(t)$, it is sampled at a rate $1 / T_{d}$ and generates the sequence $y_{a}, a=-L_{l}, \ldots, N_{c}-1$. The removal of ISI in the sequence is given by, $a=0 \ldots, N_{c}$ -1 and it has been demodulated by exploiting fast fourier transform (FFT). The output of the FFT demodulated sequence is

$$
R_{n}=\sum_{a=0}^{N_{c}-1} y_{u} e^{-j 2 \pi n a / N_{c}}, \quad n=0, \ldots, N_{c}-1 .
$$

where, $R_{n}$ be the output of the FFT demodulated sequence, $N_{c}$ be the complex-valued symbols. Further, assuming that the fading on each sub-channel is flat, a received signal $R_{n}$ is represented in frequency domain accordingly,

$$
R_{n}=H_{n} S_{n}+N_{n}, \quad n=0, \ldots, N_{c}-1
$$

where $H_{n}$ be the complex-valued flat-fading co-efficient, $S_{n}$ is the complex-valued source symbols and $N_{n}$ represents the noise of the nth sub-channel. 


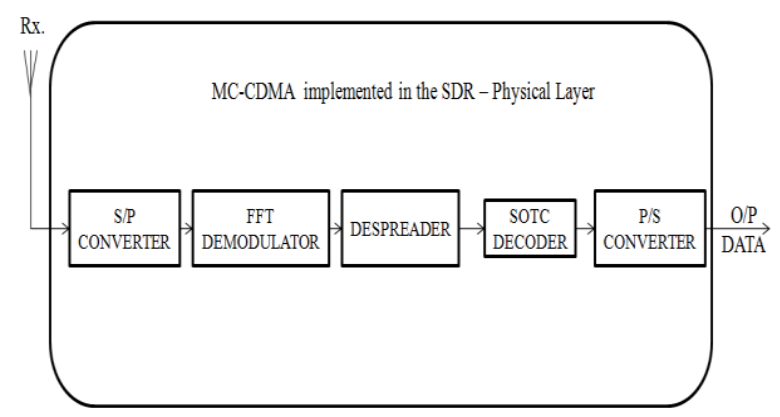

Fig-2: MC-CDMA based SDR Receiver

After serial to parallel conversion, the signal is demodulated using FFT. Then the decoding with SOTC decoder provided with the same spreading sequence used during transmission is done. Finally contented signal is received by appropriate users with high performance.

\section{TURBO CONVOLUTIONAL CODES}

Turbo codes are a refinement of concatenated encoding structure and it applies an iterative method for decoding the associated code sequence. It has been proved that turbo codes achieves a bit error probability of $10^{-5}$ using a code rate of $1 / 2$ over an additive white gaussian noise (AWGN) channel and BPSK modulation [7] at an SNR of $0.7 \mathrm{~dB}$. Turbo decoding passes the soft decision from the output of one decoder to the input of the other decoder and iterates this process several times so as to produce more reliable decisions.

\subsection{Recursive Systematic Convolutional (RSC) Codes}

RSC codes belong to the class of infinite impulse response (IIR) convolutional codes that serve as building blocks for a turbo code. It results in better error performance compared to non systematic convolutional (NSC) codes by using a feedback loop and setting one of the two outputs equal to $d_{k}$. The output $a_{k}$ is recursively calculated as follows,

$$
a_{k}=d_{k}+\sum_{i=1}^{K-1} g_{i}^{\prime} a_{k-i} \bmod 2
$$

where $d_{k}$ is the data given as input, the constraint length is $\mathrm{K}=3$, and $a_{k}$ is the output sequence. Consider a parallel concatenation of two RSC encoders; it produces code words from each component encoders. Turbo code performance is largely influenced by minimum-weight code words; it is the IIR property of RSC codes that protects against the generation of low-weight code words and thus makes turbo code more preferable for budding systems.

\subsection{Super Orthogonal Turbo Codes (SOTC)}

SOTC is a low-rate convolutional turbo code [7] that can be applied as spreading codes in MC-CDMA system. It is well suited for spread spectrum applications. The idea behind SOTC is to concatenate two component codes i.e. recursive systematic code (RSC) and a Walsh Hadamard (WH) generator. It helps to achieve good performance results for moderate users in uplink as well as in the downlink. This attempt of combining two codes is made to minimize the computational complexity [9] with more number of users.

\subsubsection{SOTC Encoder}

An orthogonal convolutional encoder consists of orthogonal output sequences for each user. If the memory of the code is $m$, there are $2^{m}$ different encoder states and a total of $2^{m+1}$ orthogonal sequences are required. Therefore, to get a set of orthogonal sequences, Walsh functions can be used. SOTC is simply a concatenation of recursive systematic code (RSC) and WH generator [14]. The inner encoder is the walsh-hadamard generator obtained from Hadamard matrices that can be generated as shown below,

$$
H_{1}=[0] \quad H_{2 i}=\left[\begin{array}{ll}
H_{i} & H_{i} \\
H_{i} & H_{i}^{\prime}
\end{array}\right]
$$

Thus Hadamard matrices can be easily generated from (11). Fig-3 depicts an example of a SOTC encoder with memory $m=3$.

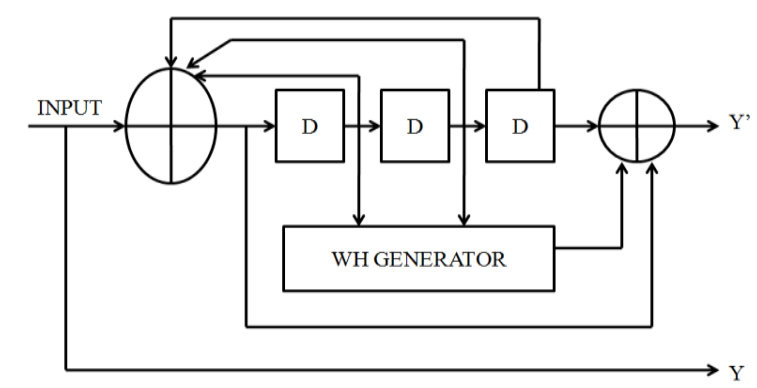

Fig-3: Super Orthogonal Turbo Encoder

The initial data sequence is encoded using RSC code whose systematic output and interleaved output is given as input to the inner encoder i.e. WH generator. WH generator can simply be a look-up table that stores the output sequences [14]; it sends the signal for modulation. Thus the input data is coded using SOTC code and modulated using IFFT and transmitted through the channel.

\subsubsection{SOTC Decoder}

After transmitting the signal over a fading or AWGN channel, the received signal is represented as $\mathrm{Y}$ and $\mathrm{Y}^{\prime}$, where $\mathrm{Y}$ is the systematic output and $\mathrm{Y}^{\prime}$ is the parity output. The channel outputs are

$$
y_{t}^{i}=A_{t}^{i}\left(2 x_{t}^{i}-1\right)+\gamma_{t}^{i}
$$

where $\gamma_{t}^{i}, i=0,1, \ldots, n-1$ is Gaussian random variables with equal variance. In general, $A_{t}^{i}=1$ for all $i$ and $t$ in an AWGN channel.

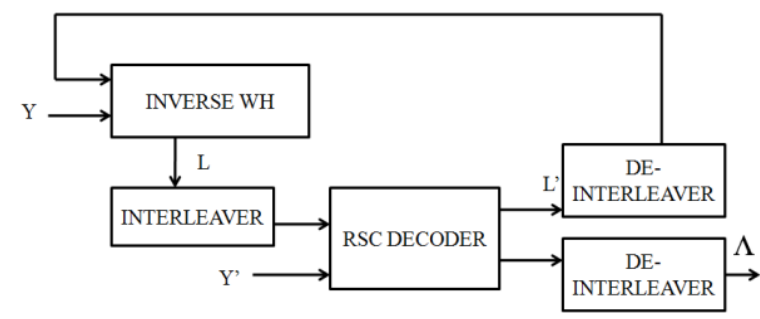

Fig-4: Super Orthogonal Turbo Decoder 
The channel outputs $\mathrm{Y}$ and $\mathrm{Y}^{\prime}$ are decoded using inverse Walsh-Hadamard (IWH) and RSC decoder. The output from IWH is interleaved and sent to inner decoder for iterative decoding. A requirement for iterative decoding is the a priori information provided by the previous decoder must remain independent from the other inputs. This is accomplished by interleaving the data sequence before it enters the second component decoder. $\Lambda_{t}$ represents the soft decision made by the decoder called log-likelihood ratio which is defined as,

$$
\Lambda_{t}=\log \frac{P(\text { input }=1 \mid \text { observation })}{P(\text { input }=0 \mid \text { observation })}
$$

The log-likelihood ratio estimate [14] of a data bit can be expressed as the sum of

$$
\Lambda_{t}=L_{t}^{(a p r)}+L_{t}^{(e x t)}
$$

From Fig- $4, L_{t}^{(a p r)}$ is the a priori estimate provided by the previous decoder. The next term, $L_{t}^{(e x t)}$ represents the extrinsic information provided by the current decoder, which in turn will be used as a priori information by the next decoder. The number of iterations determines the performance of decoder. The iterations can be completed with a predefined value or if a fixed threshold value has been reached.

\subsection{Turbo Decoder}

A turbo decoder needs to generate the a posteriori probability (APP) or soft decision output for each decoded bit. Unfortunately, the viterbi algorithm is not suited to generate the APP. The Bahl algorithm was proposed for decoding RSC codes. The APP of a decoded data bit $d_{k}$ can be derived from the joint probability $\lambda_{k}^{i, m}$ defined by,

$$
\lambda_{k}^{i, m}=P\left\{d_{k}=i, S_{k}=\left(m \mid R_{1}^{N}\right)\right\}
$$

Where $S_{k}$ is the encoder state at time $k$ and $R_{1}^{N}$ is a received binary sequence from time $k=1$ through some time $N$. Thus, the APP for a decoded data bit $d_{k}$, represented as a binary digit, is equal to

$$
P\left\{d_{k}=i \mid R_{1}^{N}\right\}=\sum_{m} \lambda_{k}^{i, m} \quad i=0,1
$$

The log likelihood ratio (LLR) is defined as the logarithm of the ratio of APPs, as follows

$$
L\left(\widehat{d_{k}}\right)=\log \frac{\sum_{m} \lambda_{k}^{1, m}}{\sum_{m} \lambda_{k}^{0, m}}
$$

Further, maximum a posteriori (MAP) decision rule is applied by comparing $L\left(\widehat{d_{k}}\right)$ to a zero threshold and decision is made by the decoder. The conditions for comparison is as follows,

$$
\begin{aligned}
& \widehat{d_{k}}=1 \text { if } L\left(\widehat{d_{k}}\right)>0 \\
& \widehat{d_{k}}=0 \text { if } L\left(\widehat{d_{k}}\right)<0
\end{aligned}
$$

\section{TURBO MULTIUSER DETECTION (MUD) TECHNIQUES}

Detection techniques can be classified as either single-user detection or multi-user detection (MUD). Generally, single-user detection is not preferred in
MC-CDMA system since it is sub-optimal. It detects the user signal of interest by not taking into account any information about multiple access interference and therefore it is sub-optimal. This has been rectified in multi user detection by using a priori knowledge of the spreading codes but at the expense of receiver complexity. The problem of MAI and remedies to overcome are explained below.

\subsection{Multiple Access Interference (MAI)}

In MC-CDMA system, the spreaded signal is passed through a frequency selective channel to the receiver. When the received codes are not orthogonal to each other, in other words, if the cross-correlation is non-zero, there arises multiple access interference (MAI). There are many techniques to overcome MAI among which multi user detection technique [11] is well suited for MC-CDMA systems. The design of spreading codes is considered to remove the effects of MAI completely [12]. In order to transmit MAI free signals, a basic concept is explained. Consider a Walsh-Hadamard (WH) sequence of length $M$, say $W H=d_{k} ; k=1,2, \ldots, M$. Each user transmits a sequence of $M$ samples which are uniformly spaced at a distance $R$. These $M$ samples are obtained by spreading the data by a specified element of $W H$ sequence. The calculated frequency domain sequence as follows,

$$
c_{k}(i)=\frac{1}{\sqrt{N}} \sum_{m=0}^{M-1} d_{k}(m) e^{-j \frac{2 \pi}{M}(i m)} \quad 0 \leq i N-1 .
$$

where $c_{k}(i)$ be the spreaded signal without MAI, $M$ is the different codes used for spreading and $i$ is the user.

\subsection{Log-MAP Algorithm}

For any kind of iterative decoding algorithms, the basic tool is the log likelihood ratio (LLR). It is defined as the ratio between the required data and the transmitted data. For a convolutional code, the LLR information about sequences is calculated using MAP receiver. The MAP algorithm [13] also known as BCJR algorithm provides the exact LLR value for a bit rather than an approximate one, but this is achieved at the expense of complex detection. The LLR ratio is written as,

$$
\wedge_{1}\left(d_{k}\right)=\log \frac{\operatorname{Pr}\left(d_{k}=\left(1 \mid R_{1}^{N}\right)\right)}{\operatorname{Pr}\left(d_{k}=\left(0 \mid R_{1}^{N}\right)\right)}
$$

$\operatorname{Pr}\left(d_{k}=\left(1 \mid R_{1}^{N}\right)\right.$ is the a posteriori probability (APP) of the information input data at time $\mathrm{k}\left(d_{k}\right)$ when it is equal to 1 given the entire received data. $R_{1}^{N}$ is the block data sequence and the state of the encoder $S_{k}$ is represented as,

$$
S_{k}=\left(a_{k}, a_{k-1}, \ldots \ldots, a_{k-v+1}\right)
$$

where $a_{k}$ is the output of the first shift register in the RSC encoder. The APP of $d_{k}$ is thus equal to

$$
\operatorname{Pr}\left(d_{k}=\left(j \mid R_{1}^{N}\right)\right)=\sum_{s} \Gamma_{k}^{j}(s), \quad j=0,1
$$

The MAP algorithm defines the joint probabilities of in terms of three parameters,

$$
\alpha_{k}=\operatorname{Pr}\left(\mathrm{S}_{\mathrm{k}}=\mathrm{s} \mid \mathrm{R}_{1}^{\mathrm{k}}\right)
$$




$$
\begin{gathered}
\beta_{k}(s)=\frac{\operatorname{Pr}\left(R_{k+1}^{N} \mid S_{k}=s\right)}{\operatorname{Pr}\left(R_{k+1}^{N} \mid R_{1}^{k}\right)} \\
\gamma_{j}\left(R_{k}, s^{\prime}, s\right)=\operatorname{Pr}\left(d_{k}=j, S_{k}=s, R_{k} \mid S_{k-1}=s^{\prime}\right)
\end{gathered}
$$

The LLR is now described in terms of these parameters as,

$$
\Lambda_{1}\left(d_{k}\right)=\log \frac{\sum_{s} \sum_{s^{\prime}} \gamma_{1}\left(R_{k}, s^{\prime}, s\right) a_{k-1}\left(s^{\prime}\right) \beta_{k}(s)}{\sum_{s} \sum_{s^{\prime}} \gamma_{0}\left(R_{k}, s^{\prime}, s\right) a_{k-1}\left(s^{\prime}\right) \beta_{k}(s)}
$$

\section{SIMULATION RESULTS}

The proposed system is simulated using MATLAB version 7.14.0. The frame size for RSC codes is 518 and for SOTC it is generally in multiples of 2 . The transmission takes place through additive white gaussian noise (AWGN) channel for both the systems since it shows improved performance. The parameters required to execute the code is specified in table-1 shown below.

Table-1: Parameter Specifications

\begin{tabular}{|l|l|}
\hline \multicolumn{1}{|c|}{ PARAMETER } & \multicolumn{1}{c|}{ SPECIFICATION } \\
\hline Codes used & $\begin{array}{l}\text { Super Orthogonal Turbo } \\
\text { Codes (SOTC) and } \\
\text { Recursive Systematic Codes } \\
\text { (RSC) }\end{array}$ \\
\hline Code rate used & $1 / 2$ and $1 / 3$ \\
\hline Frames Size & 512 and 1024 \\
\hline SNR & $\begin{array}{l}1 \text { to } 3(\mathrm{~dB}) \text { for code rate } 1 / 2 \\
\text { and } 1 / 3\end{array}$ \\
1 to 6 (dB) for code rate $1 / 2$ \\
\hline No. of Iterations & $5,10,15$ and 20 \\
\hline Channel & AWGN \\
\hline Interleaver & Punctured and Un punctured \\
\hline
\end{tabular}

The performance curves for different iterative values is made and shown from Fig-5 to 13 below. The two different frame size chosen are 512 and 1024 respectively. The signal to noise (SNR) ratio is given as $3 \mathrm{~dB}$ for all the iterative values. The graph is plotted for number of iterations along $\mathrm{x}$-axis and bit error rate (BER) along $\mathrm{y}$-axis for all the following outputs.

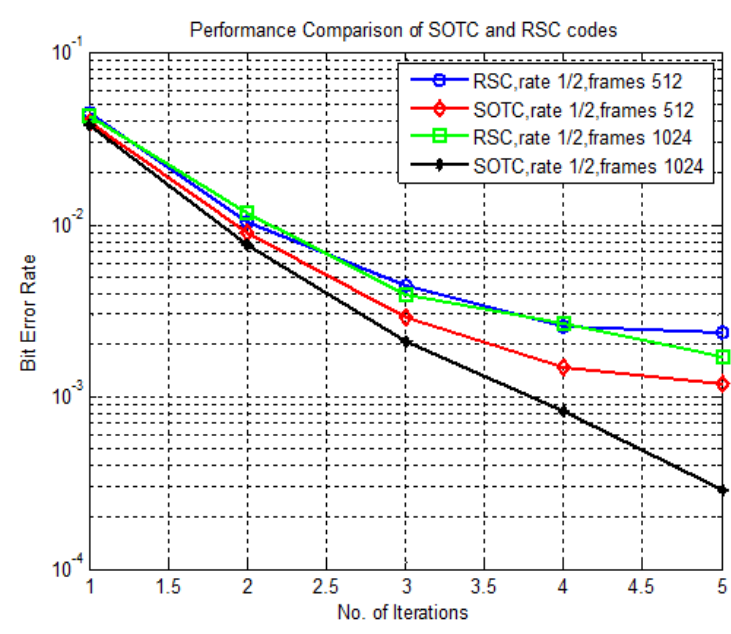

Fig-5: BER performance of MC-CDMA based SDR system for 5 iterations

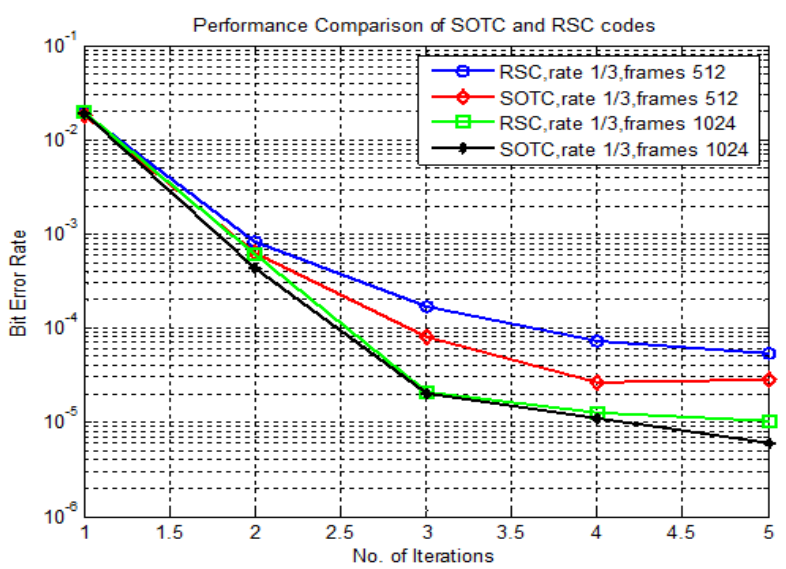

Fig-6: BER performance of MC-CDMA based SDR system for 5 iterations

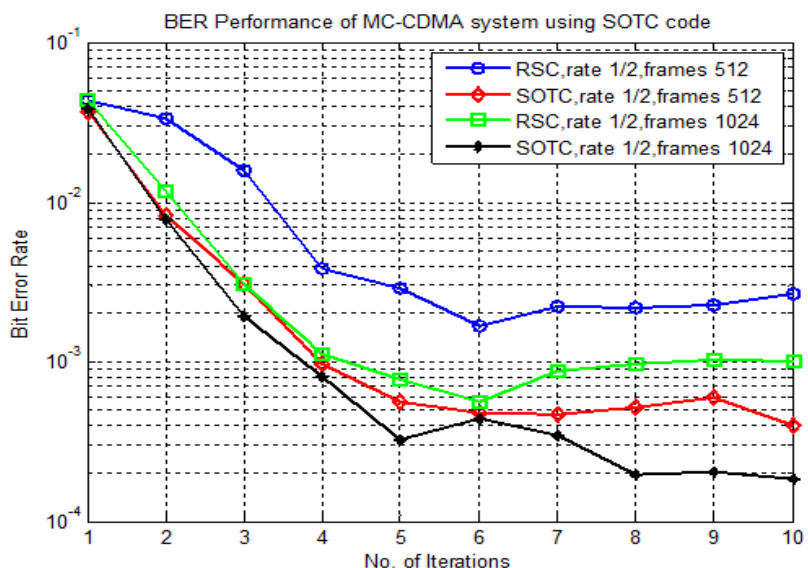

Fig-7: BER performance of MC-CDMA based SDR system for 10 iterations 


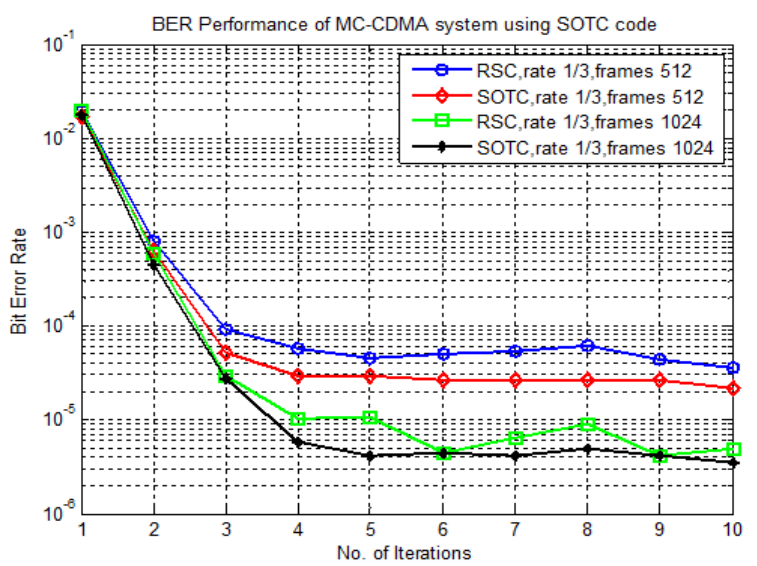

Fig-8: BER performance of MC-CDMA based SDR system for 10 iterations

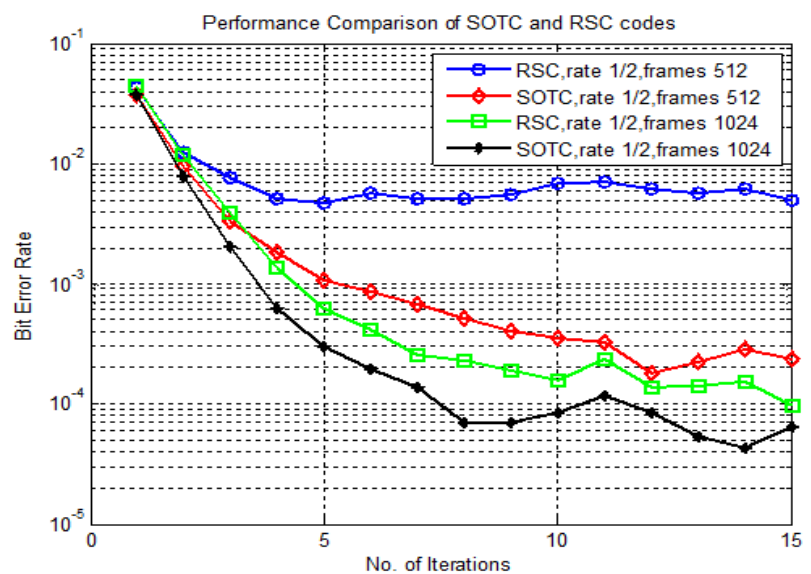

Fig-9: BER performance of MC-CDMA based SDR system for 15 iterations

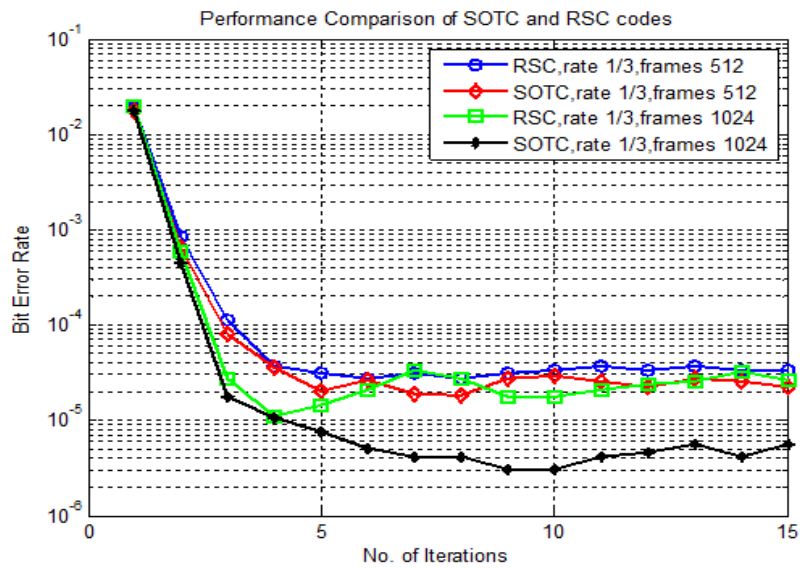

Fig-10: BER performance of MC-CDMA based SDR system for 15 iterations

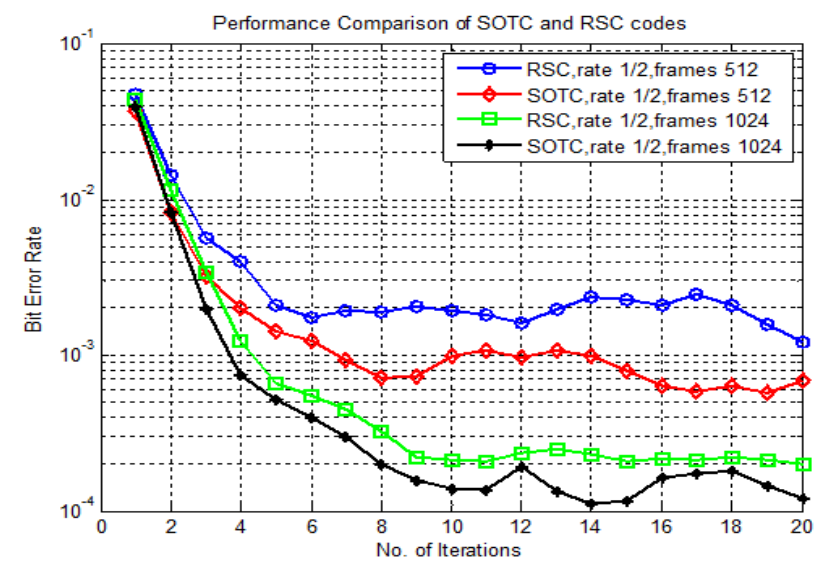

Fig-11: BER performance of MC-CDMA based SDR system for 20 iterations

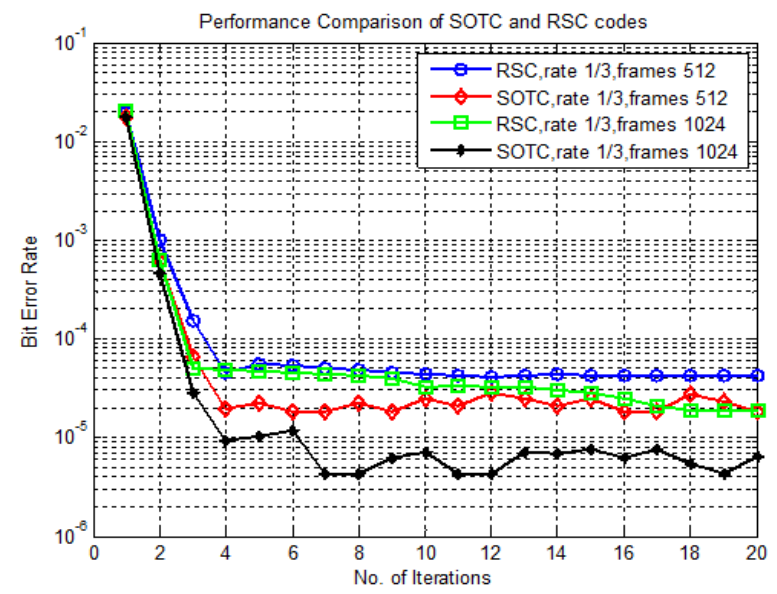

Fig-12: BER performance of MC-CDMA based SDR system for 20 iterations

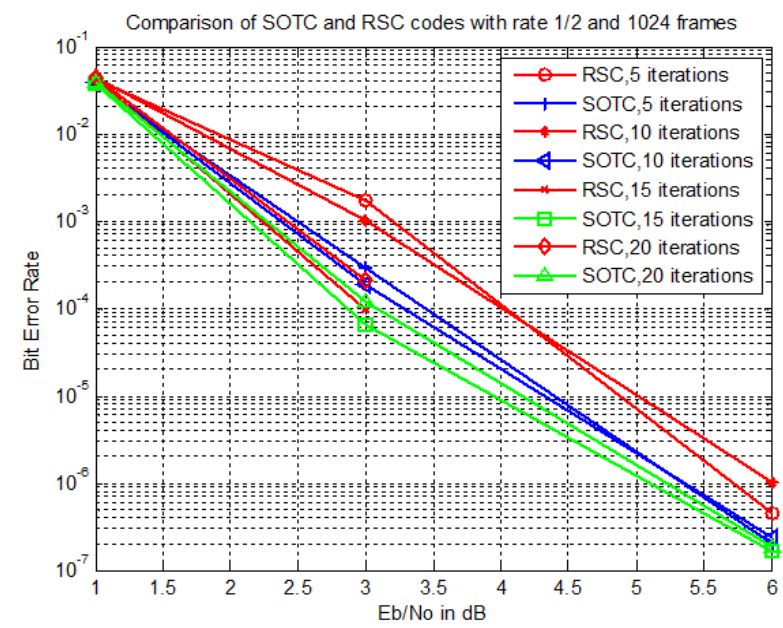

Fig-13: BER performance of MC-CDMA based SDR system 
From the results obtained, the performance of MC-CDMA based SDR using SOTC is outstanding as the number of iterations increases and it outperforms RSC codes. Table- 2 shows the improved range in numerical values as follows,

Table-2: Performance Comparison of MC-CDMA system using SOTC and RSC codes

\begin{tabular}{|c|c|c|}
\hline No. of Iterations & $\begin{array}{c}\text { MC-CDMA } \\
\text { using SOTC code }\end{array}$ & $\begin{array}{c}\text { MC-CDMA } \\
\text { using RSC code }\end{array}$ \\
\hline 5 & 0.0004293 & 0.00206 \\
\hline 10 & 0.0001305 & 0.0003472 \\
\hline 15 & $9.148 \mathrm{e}-005$ & 0.0002777 \\
\hline 20 & $9.707 \mathrm{e}-005$ & 0.000127 \\
\hline
\end{tabular}

\section{CONCLUSION}

MC-CDMA based SDR system using super orthogonal turbo code with log-MAP algorithm is proposed in this paper. Simulation is done using MATLAB version 7.14.0. The results show that the proposed system outperforms MC-CDMA using RSC codes. The simulations are made with various iterative values for comparison of both systems. Its observed that as the number of iterations increases, the performance in terms of BER is excellent using SOTC with $\log$-MAP algorithm. This improvement is more evident from the outputs obtained over AWGN channel. Thus completely removing the effects of MAI and mitigating complex detection to a great extent, the proposed system would be preferable for next generation wireless communication. In future, complex detection can further be reduced when the number of users increases by using combinations of concatenated turbo codes and efficient decoding algorithms.

\section{REFERENCES}

[1] Faouzi Bader, Labed Nadjet and Ismael Gutierrez, 'Intercell Interference Investigation in a MC-CDMA System with Iterative Demapping', IEEE Vehicular Technology Conference, pp. 1-5, September-2006.

[2] M.Saravanan and S.Ravi, 'Performance Analysis of Different Spreading Codes in CDMA System Environment for Multi-User Adaptive SDR Environment,' IJCSNS International Journal of Computer Science and Network Security, vol.11, no.7, pp. 28-37, July-2011.

[3] K.Rasadurai, J.Dhanancheziyan, K. Pushpakodi, N. Kumaratharan, 'Performance enhancement of MCCDMA systems through MAP based Multiuser Detection,' International Journal on Advanced Networking and Applications, vol. 3, no. 6, pp. 14381443, April-2012.

[4] Junqiang Li, Khaled Ben Letaief and Zhigang Cao, 'Reduced-Complexity MAP-Based Iterative Multiuser Detection for Coded Multicarrier CDMA Systems,' IEEE Transactions on Communications, vol.52, no.11, pp. 1909-1915, November-2004.
[5] K. Rasadurai and N. Kumaratharan, 'Performance Enhancement of Asynchronous MC-CDMA system through Turbo MUD,' International Research Journal of Mobile and Wireless Communication (IRJMWC), vol.3, no.1, pp.89-94, January-April 2012.

[6] Petri Komulainen and Kari Pehkonen, 'Performance Evaluation of Super Orthogonal Turbo Codes in AWGN and Flat Rayleigh Fading Channels,' IEEE Journal on Selected Areas in Communications, vol.16, no.2, pp. 196-205, February-1998.

[7] Durai Thirupathi and Keith M. Chugg, 'A Simple Construction of Low rate Convolutional codes with application to Low rate Turbo-like Code Design,' Global Telecommunications Conference, vol. 2, pp. 1305-1309, November-2002.

[8] F. Portiere et. al., ' Performance Comparison of Multiuser Detectore for the Downlink of a Broadband MCCDMA system, 'Proceeding IST Mobile \& Wireless Communications Summit, ' arXiv:cs/0606117, June-2006.

[9] Shaikh Enayet Ullah and Md. Mahbubar Rahman, 'BER Performance Analysis of a FEC Encoded Multi-user MIMO MCCDMA Wireless Communication System,' International Journal of Hybrid Information Technology, vol. 4, no. 3, pp. 41-50, July-2011.

[10] Ali Reza Enayati et. al., 'A Novel Bandwidth Efficient SOC-based Turbo coding scheme Mid Reduced Complexity MUD for SA-based MC-CDMA systems,' Telecommunication Systems, vol. 5, no. 2, pp. 71-88, 2012.

[11] Jaswinder Singh, 'Techniques for Reduction of Multiple Access Interference in Fiber-Optic CDMA Systems,' Journal of Engineering Research and Studies, E-ISSN 976:7916.

[12] Kaiser, Stefan and Joachim Hagenaeur, 'Multi-Carrier CDMA with Iterative Decoding and Soft-Interference Cancellaiton,' Global Telecommunications Conference, vol.1, pp. 6-10, November-1997.

[13] Lucian Andrei Perisoara and Rodica Stoian, 'The Decision Reliability of MAP, log-MAP, Max-log-MAP and SOVA Algorithms for Turbo Codes,' International Journal of Communications, vol. 2, no. 1, pp. 65-74, June-2008.

[14] Petri Komulainen and Kari Pehkonen, 'Performance Evaluation of Super Orthogonal Turbo Codes in AWGN and Flat Rayleigh Fading Channels,' IEEE Journal on Selected Areas in Communications, vol.16, no.2, pp. 196-205, February-1998.

\section{Authors Biography:}

T. Ilavarasi received her B.Tech in Electronics and Communication Engineering from Pondicherry University, Pondicherry, in 2011. Currently she is pursuing Master of Engineeringin Computer and Communication from Sri Venkateshwara College of Engineering, Anna University, Chennai. Her area of interest includes Wireless Communication, MC-CDMA systems and Multiuser detection techniques. 
N. Kumaratharan received B.E degree in Electrical and Electronics Engineering from the University of Madras in 2001 and M.E degree in Applied Electronics from College of Engineering, Guindy, in 2004 and a Ph.D in Wireless Communication from Pondicherry Engineering College in 2010. Currently he is working as Associate Professor, Department of Information Technology; Sri Venkateshwara College of Engineering, Sriperumbudur. He has published fifteen papers in International Journals and presented fourteen papers in IEEE International Conferences and a reviewer of some International Journals and Conferences. $\mathrm{He}$ is a recognized $\mathrm{Ph} . \mathrm{D}$ Supervisor of Anna University, Chennai and is currently guiding seven research scholars. His area of interest includes Wireless Broadband Communication and Spread Spectrum Techniques.
K. Rasadurai received his B.E degree in Electronics and Communication Engineering from Anna University, Chennai in 2006. He received his M.E Degree in Embedded System Technologies from Anna University, Chennai in 2009. He has three and half years of teaching experience. He is presently pursuing his research in the area of wireless mobile communication. He has published three papers in International Journals and presented four papers in International conference. His area of interest includes mobile communications, wireless network 